\section{Sines in terse verse}

\section{Coding large numbers in synthetic words made them easier to memorize.}

\section{Roddam Narasimha}

$\mathrm{H}$ ere is a bizarre jumble of sounds and/ or words - not, as one might think, the rhythm syllables that accompany an Indian dance, but a piece from a renowned Sanskrit work on mathematics and astronomy:

makhi bhakhi phakhi dhakhi nakhi ñakhi nakhi hasjha skaki kisga ghakhi kighva | ghlaki kigra hakya dhaki kica

sga s'jha inva kla pta pha cha kala-ardha-jyāh \|

It is verse 12 from the $\bar{A}$ rya-bhatiya written by the great Indian astronomermathematician Äryabhata in 499 CE. The sounds are strange not because the language maybe unfamiliar; in fact only the last phrase in the verse contains words that you can find in a dictionary of classical Sanskrit.

The verse is effectively a table of sines, and each of the 24 sound-bytes preceding the last phrase is a synthetic word that represents a number, according to a code devised by Äryabhata and explained at the beginning of the book. The code was an ingenious solution to the problem of writing mathematics in terse verse. Verse was the norm in Sanskrit writing, even in science, for it was long before the days of paper and printing, and it had to be terse so that it could be memorized without being an undue burden. Some of these verses are so cryptic that their meaning becomes apparent only from the much longer commentaries - often written in prose - that are the other part of the tradition of Sanskrit writing. A rryabhata was such a powerful and seminal source of tool and thought in Indian astronomy and mathematics that his work attracted a large number of very distinguished commentaries among the last being one written as late as the nineteenth century by Kodanda-rama. It is also possible that the 'terse verse' ensured that the intellectual property did not get lost by falling into whatever was considered 'unauthorized' or 'undeserving' hands.

In Āryabhata's system, the 25 'classified' consonants of the Sanskrit alphabet, $k$ to $m$, stand for the numbers 1 to 25 ; the eight unclassified consonants $y$ to $h$ stand for the numbers 30 to 100 in steps of 10 . The place value is indicated by the nine vowels $a$ to $a u$, (counting long and short as equivalent) progressively from $100^{\circ}$ to $100^{8}$ in steps of 100 (as the consonants cover 1 to 100 , only powers of 100 are needed). A number is denoted by a simple or compound consonant with a vowel, or a string of such sound-bytes: a compound consonant denotes the sum of the individual ones. Thus $k a=1, k i=100$ ( $i$ is the second vowel and stands for $10^{2}$ ), $g u=30,000$ ( $g$ stands for $3, u$ is the third vowel), $g n u=23 \times 100^{2} \quad(n$ is for 20, $g n=3+20$ ), and so on. Huge numbers can in this system be represented by short, synthetic words; for example, khyughr= $\left(4 \times 100^{3}\right)+(2+30) 100^{2}=4,320,000$, which was Āryabhata's postulate for the total number of eastward revolutions of the Sun during an Indian epoch known as yuga.

Going back to the sines, I must hasten to clarify that the entries in Āryabhata's verse more precisely provide a set of differences of half-chords for a given radius. As the values are given for 24 divisions of a quadrant, the incremental angle is 3.75 degrees, or 225 minutes; the first difference is makhi= $\left(25 \times 100^{\circ}\right)+(2 \times 100)=225$, which is of course sin 225 minutes $-\sin 0$, normalized to 225 . The actual sines can be obtained by adding up the differences; Âryabhata's values are always correct to three significant figures, very often four. The use of half-chords, rather than the full chord as in Greek practice, was crucial to the emergence of the modern sine (which differs from Āryabhata's in being normalized to the interval 0 to 1 ). It is not clear why Āryabhata preferred to give differences instead of the sines themselves; perhaps it was because they yielded to a more compact versification, or because they were necessary for extending the tables, or because they helped in interpolation: after all, in $665 \mathrm{CE}$ Brahmagupta offered (in a book called Khanda-khädhyaka, literally The [mathematical] $\dot{S}$ weet Eat) a second-order interpolation formula corresponding to Newton-Stirling; and it is known that classical Indian mathematicians used the 'smooth' variation of differences as a criterion in the final selection of listed values for the function (amusingly, there are copies of the text that have the sines right but the metre is flawed).

Although the use of letters to indicate

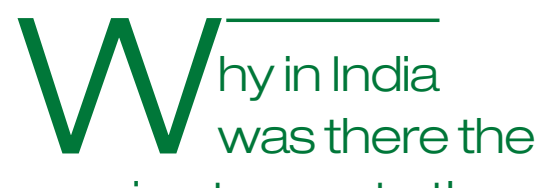
passion to create the shortest phrases for huge numbers?

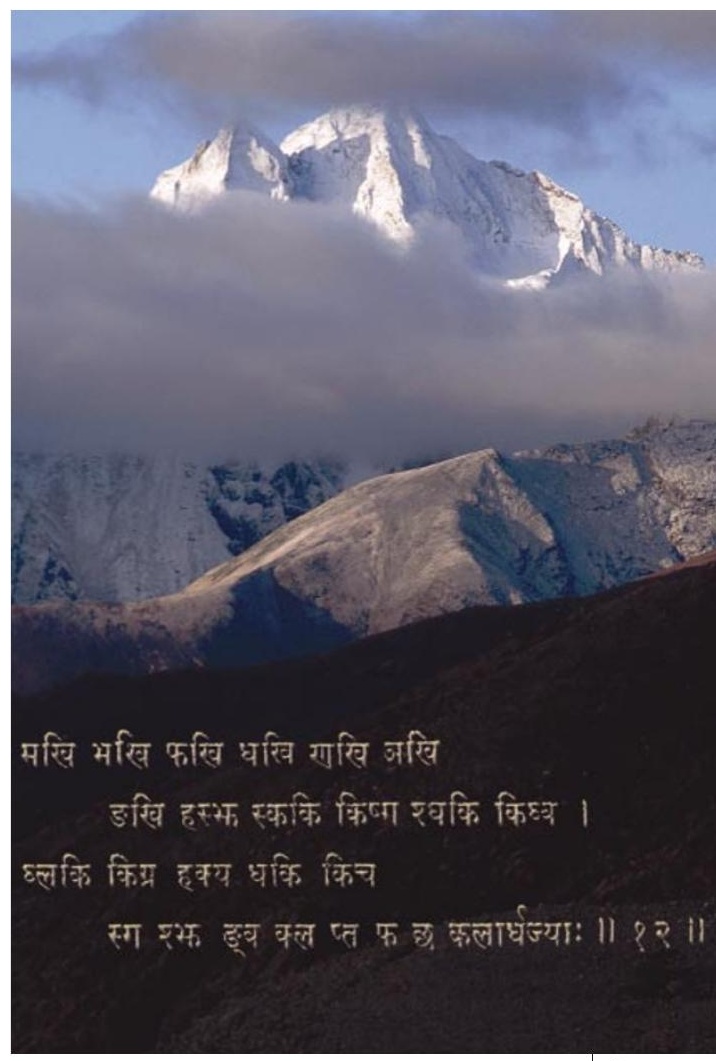

Sine language: this Sanskrit is a numerical table.

numbers was widespread in the world, most familiarly in Greek and Roman cultures, the Āryabhata system was fundamentally different in two respects. First, the system implicitly recognized the decimal place-value principle, for the rank of the vowel determined an appropriate power of 10 . Second, the combination of consonants and vowels made pronounceable synthetic words, which could be integrated into text and metre.

There were other less ingenious syllabic systems in use in India, but without going into them here, we can raise some interesting questions. Why, for example, was there no dread of large numbers in Indian culture? Did familiarity with $10-\mathrm{km}$-high mountains and kilometres-wide rivers flowing thousands of kilometres across vast plains to huge oceans have something to do with it? And why was there, at the same time, the passion to create the shortest phrases for those huge but friendly numbers? It was said of Indian grammarians that if they could save even half a syllable from one of their rules, they celebrated it like the birth of a son. Sound was holy, and a syllable could hold the infinite: verbal minimalism appears to have gone with numerical opulence.

Roddam Narasimha is director of the National Institute of Advanced Studies, Indian Institute of Science Campus, Bangalore 560 012, India.

\section{FURTHER READING}

Shukla, K. S. \& Sarma, K. V. Ārya-bhatīya of Āryabhata (Indian National Science Academy, New Delhi, 1976). Yano, M. Indian J. Hist. Sci. 12, 83-89 (1977). 